\title{
Parent-offspring conflict and transition to crèche phase in Chinstrap Penguins (Pygoscelis antarctica)
}

\author{
Virginia Morandini ${ }^{1} \cdot$ Javier Viñuela $^{2} \cdot$ Josabel Belliure $^{3} \cdot$ Miguel Ferrer $^{4}(\mathbb{D}$
}

Received: 17 December 2019 / Revised: 11 July 2021 / Accepted: 9 August 2021 / Published online: 19 August 2021

(c) The Author(s) 2021

\begin{abstract}
Parent-offspring conflict theory predicts that offspring seek to prolong the parental care period, while parents strive to shorten it as much as possible. Transition to the crèche phase in penguins involves changes in the dynamic of parental investment in chicks, and thus may be modulated by interests that benefit parent or offspring. We explored parent-offspring interactions in the Chinstrap Penguin (Pygoscelis antarctica) to understand the influence such interactions would have on transition to crèche phase. We studied the effect of hatching date, nestling age, brood size, and nest location on parent-offspring interactions and the length of the guard phase. All behavioral variables related to nestling demands for parental investment increased as well as parental aggression towards their chicks, with increased nestling age. Brood size showed significant effects on both nestling and parental behavior. Hatching date was negatively correlated with the age at crèche. Consequently, the decision to leave the chicks unguarded seemed to be more based on the condition of adults rather than of the chicks. Given the short time available for breeding in Antarctica and that penguins are long-lived birds with several opportunities to breed, there may be conflicting selective factors between investment in feeding chicks versus extending the period of resource acquisition and storage prior to moult.
\end{abstract}

Keywords Pygoscelis antarctica $\cdot$ Dependence period $\cdot$ Parental investment $\cdot$ Guard to creche transition $\cdot$ Antarctica $\cdot$ Deception Island

\section{Introduction}

According to parent-offspring conflict theory (Trivers 1974; 1985), offspring are selected to prolong the parental care period, while parents are selected to cease investing in offspring once the cost of parental care exceeds the benefits obtained in terms of lifetime reproductive success. In birds,

Miguel Ferrer

mferrer@ebd.csic.es

1 Oregon Cooperative Fish and Wildlife Research Unit, Department of Fisheries and Wildlife, Oregon State University, 104 Nash Hall, Corvallis, OR 97331, USA

2 Instituto de Investigación en Recursos Cinegéticos, IREC (CSIC, UCLM, JCCM), Ronda de Toledo s/n, 13005 Ciudad Real, Spain

3 Department of Life Sciences, Universidad de Alcalá, 28871 Madrid, Spain

4 Applied Ecology Group, Doñana Biological Station EBD-CSIC, Avd. Americo Vespucio n. 26, 41092 Sevilla, Spain parent-offspring conflict is be expected regarding both the level of parental investment that offspring should receive and the duration of parental investment during the post-fledging period. Parent-offspring conflict during the post-fledging period has been documented for several species of birds (Bustamante and Hiraldo 1990; Ferrer 1992; Bustamante 1994; Riou et al. 2012; Watson et al. 2012; Muriel et al. 2015; Trillmich et al. 2016; López-Idiáquez et al. 2018; Davis et al. 2019). In these studies, parents progressively reduced offspring investment, promoting independence, while offspring demanded the same or more investment. For example, family break-up among raptors takes place after a period of parent-offspring conflict, with the parents promoting the juvenile independence via a gradual reduction in food supplied or even increased aggression toward offspring (Ferrer 1992).

The dependence period in several species of seabirds, including penguins, shows two markedly different stages: guard phase and post-guard or crèche phase. During the guard phase, one of the adults protects the nestlings at the nest while the other is foraging at sea; therefore, males and 
females alternate periods of fasting and foraging activity. During the crèche phase, both parents can be foraging at sea while leaving their offspring alone in a crèche. Crèching, the close association of post-guard-phase chicks, occurs in many penguin species (see Davis 1982 for review). A number of adaptive advantages have been proposed to explain the tendency for chicks to form crèches; these include protection against predation and huddling to stay warm in cold weather or even protection against aggressions by other adults (Seddon and Heezik 1993; Penteriani et al. 2003; Wilson 2009). Chinstrap Penguin (Pygoscelis antarctica) chicks, as in many other penguin species, form crèches, a habit believed to offer protection from predators such Skuas (Catharacta maccormicki) or defense against non-related adult aggressions (Penteriani et al. 2003).

Several environmental factors can affect the length of the guard phase (Jennings 2015). For example, penguins may left their chicks in crèche synchronously and take advantage of predator swamping, so that most chicks are present at the same time in the crèche and thereby overcome the predator's ability to prey on every chick, shortening the guard phase of the later chicks (Catry et al. 2008; Rothenbach and Kelly 2012; Morandini et al. 2020). Also chick age and condition may also influence the length of brood guarding (Moreno et al. 1997; Varpe et al. 2004; Catry et al. 2006). In Chinstrap Penguins, negative consequences for those young that were left unguarded younger have been reported, having to fledge at younger ages and showing slower growth rates than chicks that entered the crèche at an older age (Viñuela et al. 1996). As far as we know there is no published information analyzing parent-offspring interactions in the Chinstrap Penguins.

The aim of the present study was to explore parent-offspring interactions to describe behavioral changes during the guard phase until transition to crèche that could clarify the relative roles of parent and offspring on the duration of the guarding period in the Chinstrap Penguins. We considered chick begging behavior, chick movements towards parents, parent food delivery and parent aggressiveness as proper parent-offspring interactions variables. Parents show mild aggression toward their own chicks when they beg for food. Such aggression usually consists of mild snapping and jabbing at the chick's bill, sometimes while backing away. If the transition from guard to crèche phase is mainly an adult decision, we can expect an increase in parent aggressiveness against their offspring as well as a significant correlation between hatching dates (surrogate of parental quality, Moreno et al 1997) and creching age. Conversely, if it is an offspring decision, we can expect some kind of behavioral changes by the chicks before crèching and a high correlation between creching age and age of the nestlings.

\section{Materials and methods}

\section{Study area and species}

The study was conducted at the Vapour Col Chinstrap Penguin nesting colony (approx. 20,000 breeding pairs) on Deception Island, South Shetlands, Antarctica $\left(63^{\circ} 00^{\prime} \mathrm{S}\right.$, $60^{\circ} 40^{\prime} \mathrm{W}$ ), during the austral summer of 1993-1994. Chinstrap Penguin is a medium-size penguin (around $4 \mathrm{~kg}$ ) with a maximum clutch size of two eggs (mean clutch size $=1.93$; ref). During the breeding season, we randomly selected and marked 164 nests with numbered sticks at the end of the incubation period. Additionally, we tagged adults with metal flipper tags (standard $34 \times 17 \mathrm{~mm}$ penguin bands, Lambournes Ltd., Solihull, UK). We sampled five sub-colonies $(32,46,25,34$, and 27 nests, respectively) with more than 150 breeding pairs each and all of them in close proximity (within $0.09 \mathrm{~km}^{2}$ ). We tried to visit nests daily before hatching, whenever harsh weather did not preclude fieldwork. As hatching is normally asynchronous in this species (modal asynchrony $=1$ day, Moreno et al. 1994), we used the date of the first hatched nestling as the brood hatching date. The length of the guarding phase in this species is between 22 and 42 days (Viñuela et al. 1996).

\section{Behavioral observations}

To investigate parent-offspring interactions during the guard phase, we made $15 \mathrm{~min}$ focal observations of randomly chosen nests, including both center and edge locations in the colony ( $n=164$ nests, total observation time 2,460 $\mathrm{min}$ ). To avoid pseudo replication, each nest was observed only once. For each nest, hatching date and number of nestlings were previously known. During focal observations, interactions by chicks toward parents were quantified based on (1) frequency of begging (i.e., number of begs over the $15 \mathrm{~min}$ period), and (2) number of approaching movements toward parents (two steps or more toward the adult), while interactions by adults toward chicks were quantified based on (1) frequency of aggressions and (2) frequency of food deliveries to chicks.

In total, we analyzed 74 one-chick nests and 90 two-chick nests, randomly distributed in central or peripheral locations in the colony. Observations were distributed between 8:30 and 19:00 h, and included nestlings from 6 to 36 days of age. We monitored 15 nests per day on average during 11 working days, distributed to cover the entire guard phase. Additionally, for a subsample of 27 of those nests in the large sub-colony we obtained the date when nestlings integrated in crèche by visiting them each day, therefore providing the exact length in days of the guard phase and thus the crèching age. 


\section{Statistical analyses}

We used generalized linear models (GLM; Poisson distribution and log link function) to evaluate the effect of nestling age, hour of sampling as explanatory variables of the parent-offspring interactions: food deliveries, food begging, movements toward adults, and adult aggressive behavior toward their own chicks. We select Poisson distribution due to the distribution of the parent-offspring interaction variables. Given that brood size may affect chick growth (Moreno et al. 1998), it was included in the model as a fixed factor as well as nest position in the colony.

In addition, we conducted a GLM analyses (Normal distribution and log link function) with the subsample of 27 nests where length of the guard period was known, using duration of the guard phase as response variable. Nestling age and hatching date were introduced as explanatory variables,. Given that location of the nest in the colony can be associated to parental quality (Mínguez et al. 2001; Ferrer et al. 2014), it was included in the model (central or peripheral nests) together with brood size as fixed factors. Statistica 13.3 software statistical package was used to perform statistical procedures, and we used an alpha value of 0.05 to assess significance of results according Wald test.

\section{Results}

\section{Interactions from chicks towards parents}

Frequency of food begging increased significantly with nestling age (Table 1), ranging from 7.04 beg. $h^{-1}$ when 6-13 day-old to 14.12 beg.h ${ }^{-1}$ at the end of the guard phase, and was affected by brood size (Table 1; one-chick nests: 5.12 beg. $\mathrm{h}^{-1}$ versus two-chick nests: $15.00 \mathrm{beg} . \mathrm{h}^{-1}$ ). Nor sampling time neither nest position affected the frequency of food begging. Number of approaching movements by chicks to parents also increased in frequency with nestling age (with an observed range from 0.00 to $14.12 \mathrm{~h}^{-1}$, and were not affected by brood size (Table 1). The sampling time affected the movements of the chicks to the parents, being more frequent between 12:00 and 18:00 $\mathrm{h}$ than during the morning. Position of the nest also affected frequency of movements, being higher in peripheral nests than in central ones.

\section{Interactions from parents towards chicks}

Number of food deliveries significantly increased with nestling age, and were also affected by brood size (Table 1). During the first half of the guard phase, mean food deliveries was 2.08 per h, increasing to 4.8 towards the end of the guard phase. Chicks at two-chick nests received more than
Table 1 Results of the GLM analyses (GLM Poisson distribution and Log link function) of nestling age, brood size, nest location and hour of sampling effects on frequency of food deliveries, food begging, movements of chicks toward their parents and aggressions of adults toward their own chicks

\begin{tabular}{|c|c|c|c|}
\hline Effect & $d f$ & Wald Stat. & $p$ \\
\hline \multicolumn{4}{|l|}{ Food deliveries } \\
\hline Intercept & 1 & 9.923 & 0.0016 \\
\hline Nestling age & 1 & 17.144 & $<0.0001$ \\
\hline Brood size & 1 & 4.0113 & 0.0451 \\
\hline Nests position & 1 & 2.912 & 0.0879 \\
\hline Hour of sampling & 1 & 0.315 & 0.5742 \\
\hline \multicolumn{4}{|l|}{ Food begging } \\
\hline Nestling age & 1 & 29.021 & $<0.0001$ \\
\hline Brood size & 1 & 414.168 & $<0.0001$ \\
\hline Nests position & 1 & 206.901 & $<0.0001$ \\
\hline Hour of sampling & 1 & 83.878 & $<0.0001$ \\
\hline Intercept & 1 & 61.243 & $<0.0001$ \\
\hline \multicolumn{4}{|c|}{ Movements of chicks toward parents } \\
\hline Nestling age & 1 & 34.823 & $<0.0001$ \\
\hline Brood size & 1 & 154.008 & $<0.0001$ \\
\hline Nests position & 1 & 0.299 & 0.5840 \\
\hline Hour of sampling & 1 & 1.345 & 0.2461 \\
\hline Intercept & 1 & 0.829 & 0.3625 \\
\hline \multicolumn{4}{|l|}{ Adult aggressions } \\
\hline Nestling age & 1 & 6.209 & 0.0127 \\
\hline Brood size & 1 & 350.726 & $<0.0001$ \\
\hline Nests position & 1 & 183.210 & $<0.0001$ \\
\hline Hour of sampling & 1 & 44.162 & $<0.0001$ \\
\hline Intercept & 1 & 283.991 & $<0.0001$ \\
\hline
\end{tabular}

double number of food deliveries than chicks at one-chick nests (one-chick nest: 1.64 feeds. $\mathrm{h}^{-1}$ versus two-chick-nest: 5.33 feeds. $\mathrm{h}^{-1}$ ). Nor sampling time neither nest position affected the frequency of food deliveries.

Number of adult aggressive behaviors toward their own chicks increased strongly with nestling age (Table 1), changing from 1.4 up to 18.7 aggressions hour ${ }^{-1}$ towards the end of the guard phase. Again, adult aggressive attitude was affected by brood size of the nest (Table 1; one-chicknest: 1.62 adult aggressions $\mathrm{h}^{-1}$ versus two-chick-nest: 7.95 adult aggressions. $\mathrm{h}^{-1}$ ). Sampling time showed a significant effect in aggressions being more frequent in the morning that after 12:00 $\mathrm{h}$. Position of the nest also affected frequency of aggressions, being higher in central nests.

\section{Duration of the guard phase}

Brood size significantly affected the length of the guard period being longer in one-chick-nests (mean: 34.66 days) than in two-chick nests (30.40 days) (Table 2), and nest 
Table 2 Results of the GLM analysis (GLM normal distribution and Log link function) of factors affecting the length of the guard phase

\begin{tabular}{llrr}
\hline Effect & $d f$ & Wald stat & \multicolumn{1}{l}{$p$} \\
\hline Chrèching age & & & \\
Intercept & 1 & 183.115 & $<0.0001$ \\
Hatching date & 1 & 10.394 & $<0.0012$ \\
Nest location & 1 & 0.084 & 0.7713 \\
Brood size & 1 & 7.207 & 0.0072 \\
Nestling age & 1 & 1.627 & 0.2020 \\
\hline
\end{tabular}

location had no effect on duration of guard phase (Table 2). The length of the guard phase was highly affected by the hatching date (Table 2), and we checked that the relationship between both variables, i.e. between hatching date and crèching age of chicks, was negative $(r=-0.4981$; $p=0.0082$; Fig. 1), with chicks that hatched early in the season being older when integrating on crèches. Nevertheless, no effect was found of nestling age on the length of the guard phase (Table 2).

\section{Discussion}

All variables related with nestling behavior demanding parental investment increased with nestling's age during the guard phase. Food deliveries increased as well as food begging and directed movements toward parents. In all cases, the increases were higher with two-chick broods than with a single chick. Those results suggest that chicks are increasing their demand of food continuously during the guard phase. Consequently, our results do not support the length of the guard phase being mainly determined by drastic changes in behavior of the nestlings. Penteriani et al. (2003) demonstrated in the same species and in the same colony that parents in poor condition (i.e. higher levels of Urea in blood) deserted their chicks at an earlier age, giving support to the idea that as adult body condition worsens they are more likely to abandon their chicks.

Behavior of the parents showed a marked increase in aggressive attitude against their own chicks as the age of the chicks increased. Again, this increase was significantly higher in two-chick broods than in single chick nests. Aggressions were close to five times more frequent at the end of guard phase than at the beginning.

The age of entering into the crèche was strongly related to hatching date, giving support to the role of parents in determining the end of guard phase. If the nestling development and maturation were determinant for the guard phase length, we should expect a better correlation with the age of the young than with date of hatching. However, what we found is that chicks that hatched earlier in the season tend to be guarded longer than those that hatched later. In addition, parents that hatched only one chick tended to guard their chicks during a longer time. Parents with only one chick do not need to supply as much food as parents with two chicks. We did not find any effect of the location of the nest in the colony on crèching age. Our results suggest that the decision of leaving the chicks unguarded is made by the adults and is not related to the age of the chicks, in accordance with other authors (Viñuela et al.
Fig. 1 Relationship between hatching date and age of crèching or length of guard phase $(r=-0.4981$; $\left.p=0.0082, R^{2}=24.8\right)$. Hatching date $=$ number of days after the first identified hatched nest (range 1-25)

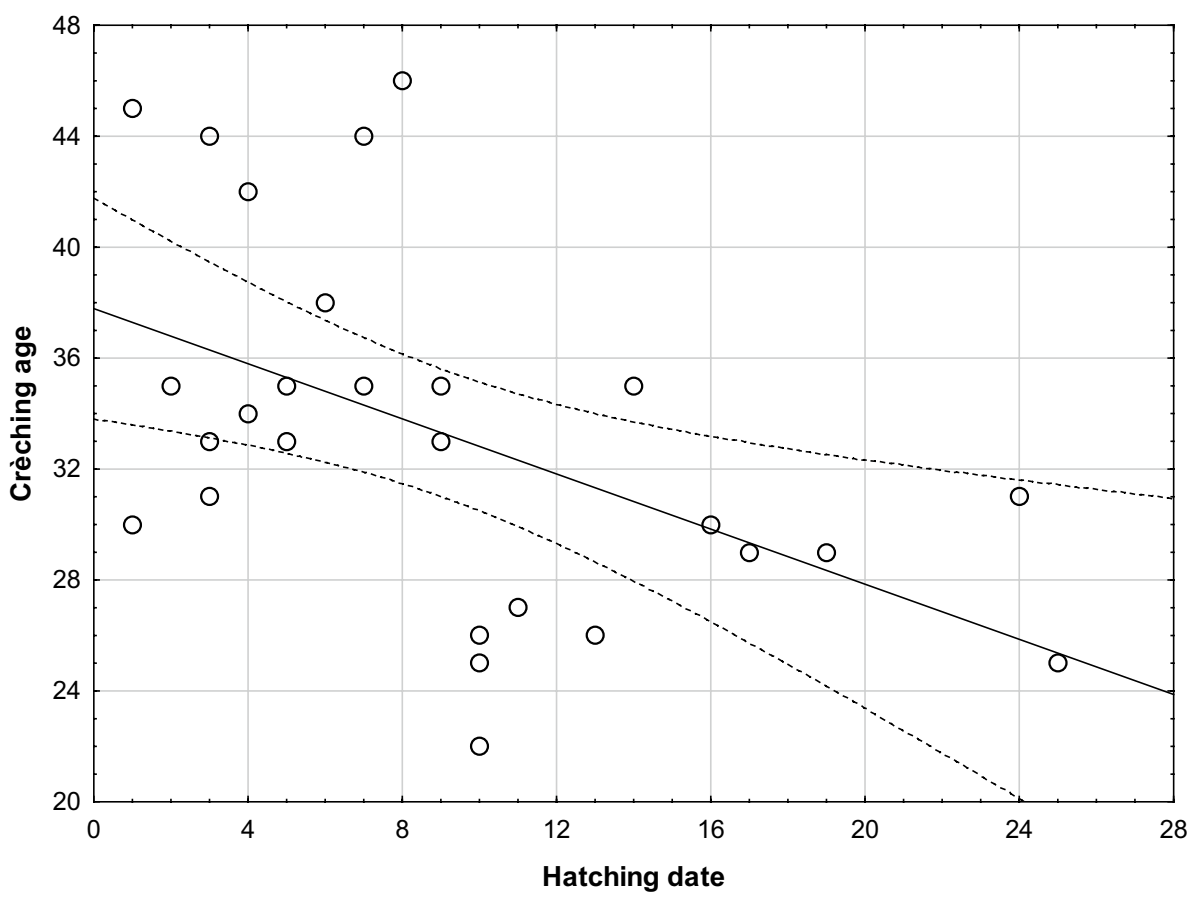


1996; Moreno et al. 1997; Williams 1990). Early laying adults leave the chicks unguarded at older ages (Moreno et al. 1997), and the decision to leave the chicks unguarded is probably based on the condition of the adults, with those in better condition and more available time before moult delaying the decision of leaving their chicks. It is interesting to note that early hatching chicks showed a high variability in creching age (from 29 to 46 days old, Fig. 1). Penteriani et al. (2003) found a similar result, arguing that first pairs breeding in a given season may be exposed to particularly high costs, because weather or foraging conditions early in the season may be worse than later on, promoting early abandonment of chicks.

After fledging of their chicks, adult penguins must moult before the end of the Antarctic summer. According to some authors (Moreno et al. 1997), there must be conflicting pressures between feeding chicks and advancing the period of premoult reserve storage, and, consequently, a clear-cut limit to the extension of the breeding season. After a critical time, they may stop the investment in the current brood to divert energy to moulting (Viñuela et al. 1996). Our results suggest that late hatching has negative consequences for chicks, owing to being left unguarded at younger ages. And earlier transition to crèche phase implies a lighter final body mass when chicks fledge and thus, a lower likelihood of survival (Viñuela et al. 1996). However, in this study we did not account for other potential factors affecting transition to crèche like nest site conditions or landscape effects (Cimino et al. 2019). Neither age nor experience of the parents, or weather variability was considered because we only have one year of data. Differences among years would be expected and differences among earlier and late breeders may be less evident in good years and accentuated in poor years (Ferrer et al. 2014).

An alternative explanation for the strong negative relationship found between hatching date and creching age would be that transition to crèche may be in part determined by the need to synchronize crèche timing for predator swamping. Nevertheless, in the island where we conducted the study, low levels of predation rates in crèche have been reported, showing that in this particular case predation does not seem a relevant factor (Penteriani et al. 2003). However, we cannot exclude the possibility that brood desertion could be, under several ecological conditions, a more flexible behavior, with adult decisions varying among years, colonies and populations.

Acknowledgements Logistic support was offered by the base Gabriel de Castilla (Spanish Army), and transport by the boat Las Palmas (Spanish Navy). Thanks to S. Jennings and two anonymous referees that greatly improved first version of this article. Ryan Baumbusch corrected the English text.
Author contributions MF conceived and designed research. MF, JV and JB and conducted field work. VM and MF analyzed data. VM wrote the manuscript. All authors read and approved the manuscript.

Funding Open Access funding provided thanks to the CRUE-CSIC agreement with Springer Nature.

\section{Declarations}

Conflict of interest The authors declared no conflict of interest in this study.

Ethical approval Procedures used in this study comply with the current laws for working in Antarctica. Permission to work in the study area and for penguin handling was granted by the Spanish Polar Committee and the SCAR. All applicable international, national, and/or institutional ethical guidelines (CSIC ethical committee) for the care and use of animals were followed.

Open Access This article is licensed under a Creative Commons Attribution 4.0 International License, which permits use, sharing, adaptation, distribution and reproduction in any medium or format, as long as you give appropriate credit to the original author(s) and the source, provide a link to the Creative Commons licence, and indicate if changes were made. The images or other third party material in this article are included in the article's Creative Commons licence, unless indicated otherwise in a credit line to the material. If material is not included in the article's Creative Commons licence and your intended use is not permitted by statutory regulation or exceeds the permitted use, you will need to obtain permission directly from the copyright holder. To view a copy of this licence, visit http://creativecommons.org/licenses/by/4.0/.

\section{References}

Bustamante J (1994) Behavior of colonial common kestrels (Falco tinnunculus) during the post-fledging dependence period in southwestern Spain. J Raptor Res 28:79-83

Bustamante J, Hiraldo F (1990) Factors influencing family rupture and parent-offspring conflict in the Black Kite Milvus migrans. Ibis 132:58-67

Catry P, Lecoq M, Strange IJ (2008) Population growth and density, diet and breeding success of striated caracaras Phalcoboenus australis on New Island, Falkland Islands. Polar Biol 31:1167-1174

Catry P, Phillips RA, Forcada J, Croxall JP (2006) Factors affecting the solution of a parental dilemma in albatrosses: at what age should chicks be left unattended? Anim Behav 72:383-391

Cimino MA, Patterson-Fraser DL, Stammerjohn S, Fraser WR (2019) The interaction between island geomorphology and environmental parameters drives Adélie penguin breeding phenology on neighboring islands near Palmer Station, Antarctica. Ecol Evol 9:9334-9349

Davis LS (1982) Creching behaviour of Adelie penguin chicks (Pygoscelis adeliae). New Zealand J Zool 9:279-285

Davis KL, Karpanty SM, Spendelow JA, Cohen JB, Althouse MA, Parsons KC, Luttazi CF (2019) Begging behavior as an honest signal of need and parent-offspring association during the postfledging dependency period. Ecol Evol. https://doi.org/10.1002/ece3.5279

Ferrer M (1992) Regulation of the period of postfledging dependence in the Spanish imperial eagle Aquila adalberti. Ibis 134:128-133 
Ferrer M, Belliure J, Minguez E, Casado E, Bildstein K (2014) Heat loss and site-dependent fecundity in chinstrap penguins (Pygoscelis antarctica). Polar Biol 37:1031-1039

Jennings S (2015) Provisioning, growth and survival of Adélie penguin chicks at Cape Crozier, Ross Island. Oregon State University, Antarctica

López-Idiáquez D, Vergara P, Fargallo JA, Martínez-Padilla J (2018) Providing longer post-fledging periods increases offspring survival at the expense of future fecundity. PLoS ONE 13:e0203152

Minguez E, Belliure J, Ferrer M (2001) Bill size in relation to position in the colony in the Chinstrap penguin. Waterbirds 24:34-38

Morandini V, Dugger KM, Ainley D, Ferrer M (2020) Rockhopper penguin-imperial cormorant mixed colonies in the Falkland islands: a stroke of luck for late breeders. Ecosphere 11:e03272. https:// doi.org/10.1002/ecs2.3272

Moreno J, Carrascal LM, Sanz JJ, Amat JA, Cuervo JJ (1994) Hatching asynchrony, sibling hierarchies and brood reduction in the Chinstrap Penguin Pygoscelis antarctica. Polar Biol 14:21-30

Moreno J, Barbosa A, Potti J, Merino S (1997) The effects of hatching date and parental quality on chick growth and creching age in the chinstrap penguin (Pygoscelis antarctica): a field experiment. Auk 114:47-54

Moreno J, Viñuela J, Ferrer M, Belliure J (1998) Food limitation in breeding chinstrap penguins (Pygoscelis antarctica): experimental evidence. J Field Ornithol 69:269-275

Muriel R, Ferrer M, Balbontín J, Cabrera L, Calabuig CP (2015) Disentangling the effect of parental care, food supply, and offspring decisions on the duration of the postfledging period. Behav Ecol 26:1587-1596

Penteriani V, Viñuela J, Belliure J, Bustamante J, Ferrer M (2003) Causal and functional correlates of brood amalgamation in the chinstrap penguin Pygoscelis antarctica: parental decision and adult aggressiveness. Polar Biol 26:538-544
Riou S, Chastel O, Hamer KC (2012) Parent-offspring conflict during the transition to independence in a pelagic seabird. Behav Ecol 23:1102-1107

Rothenbach CA, Kelly JP (2012) The parental dilemma under variable predation pressure: adaptive variation in nest attendance by Great Egrets. Condor 114:90-99

Seddon PJ, van Heezik Y (1993) Chick creching and intraspecific aggression in the jackass penguin. J Field Ornithol 64:90-95

Trillmich F, Spiller I, Naguib M, Krause ET (2016) Patient parents: do offspring decide on the timing of fledging in zebra finches? Ethology 122:411-418

Varpe $\varnothing$, Tveraa T, Folstad I (2004) State-dependent parental care in the Antarctic petrel: responses to manipulated chick age during early chick rearing. Oikos 106:479-488

Viñuela J, Moreno J, Carrascal LM, Sanz JJ, Amat JA, Ferrer M, Belliure J, Cuervo J (1996) The effect of hatching date on parental care, chick growth, and chick mortality in the chinstrap penguin Pygoscelis antarctica. J Zool 240:51-58

Watson MJ, Spendelow JA, Hatch JJ (2012) Post-fledging brood and care division in the roseate tern (Sterna dougallii). J Ethol 30:29-34

Williams TD (1990) Annual variation in breeding biology of gentoo penguins, Pygoscelis papua, at Bird Island, South Georgia. J Zool 222:247-258

Wilson D (2009) Causes and benefits of chick aggregations in penguins. Auk 126:688-693

Publisher's Note Springer Nature remains neutral with regard to jurisdictional claims in published maps and institutional affiliations. 